Annuaire suisse de politique de développement

22-2 | 2003

Société de l'information et coopération internationale

\title{
La société civile, acteur clé de la société de l'information
}

Adama Samassékou

\section{(2) OpenEdition}

1 Journals

Édition électronique

URL : http://journals.openedition.org/aspd/596

DOI : 10.4000/aspd.596

ISSN : 1663-9669

Éditeur

Institut de hautes études internationales et du développement

Édition imprimée

Date de publication : 1 novembre 2003

Pagination : 219-221

ISSN : 1660-5934

\section{Référence électronique}

Adama Samassékou, "La société civile, acteur clé de la société de l'information », Annuaire suisse de politique de développement [En ligne], 22-2 | 2003, mis en ligne le 23 mars 2010, consulté le 07 septembre 2020. URL : http://journals.openedition.org/aspd/596 ; DOI : https://doi.org/10.4000/aspd. 596

(c) The Graduate Institute / Geneva 


\title{
La société civile,
}

\section{acteur clé de la société de l'information}

\author{
Adama Samassékou*
}

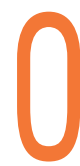

n me demande de réagir à l'article ci-dessus ${ }^{1}$ consacré à la participation de la société civile au Sommet Mondial sur la Société de l'Information (SMSI). Je le fais avec d'autant plus de plaisir qu'appartenant moi-même par ailleurs à la société civile, je me sens directement interpellé. Que quelqu'un qui est aujourd'hui activement impliqué dans les réseaux de la société civile, en Afrique et ailleurs, soit appelé à assurer les fonctions de Président du Comité préparatoire du sommet constitue en soi une symbolique très forte et surtout une heureuse opportunité pour concevoir avec l'ensemble des acteurs concernés un processus inclusif, multidimensionnel et ouvert. J'aimerais évoquer les principales caractéristiques de ce processus et apporter mon éclairage personnel sur leur signification.

\section{Un sommet d'une nouvelle génération}

Le Sommet Mondial sur la Société de l'Information, s'il s'inscrit dans la lignée des grandes manifestations de l'ONU, s'en distingue aussi à bien des égards, et je souhaiterais ici avancer l'idée d'un sommet d'une nouvelle génération.
Un sommet de l'ONU restreint aux seuls délégués gouvernementaux était, par définition, un processus intergouvernemental. C'est ce qui lui conférait jusqu'à ce jour sa légitimité et assurait (normalement) que les engagements souscrits soient respectés. Non seulement l'ouverture vers la société civile et le secteur privé apporte dorénavant au processus de négociation de plus fortes garanties en impliquant d'emblée tous les acteurs directement concernés, mais elle permet aussi de renouveler le rôle de l'Etat comme l'arbitre privilégié entre les intérêts des pouvoirs économiques et les attentes des citoyens.

Une telle ouverture nécessite des ajustements qui ne sont pas faciles à gérer d'autant que les attentes sont énormes dès que l'on admet de nouveaux acteurs à la table des négociations.

La première réunion du Comité de préparation (PrepCom) a été largement consacrée aux procédures qui devaient garantir l'établissement de relations de confiance. Les représentants de 192 Etats devaient s'entendre sur les modalités de participation des observateurs, dont font partie les 
représentants du secteur privé, des organisations intergouvernementales et, en particulier, de la société civile, constituée de milliers d'organisations qui toutes souhaitent exprimer leurs aspirations.

Tout au long du processus, les obstacles sont nombreux et rien ne sert de les occulter. Le facteur temps reste la contrainte majeure. L'ensemble des acteurs a disposé d'à peine un peu plus d'une année pour s'informer, se mobiliser, s'organiser, produire des propositions sur des sujets complexes, les défendre et entamer les négociations.

\section{Le Bureau de la société civile}

Très tôt dans le cours du processus, le besoin s'est fait sentir de structurer la participation de la société civile pour faciliter le dialogue avec les gouvernements. C'était là une condition sine qua non de la participation réelle et effective de la société civile à l'élaboration de la Déclaration et du Plan d'Action. J'ai appelé de mes vœux la création d'un tel mécanisme nécessaire à l'établissement d'un climat de confiance mutuelle. Sous l'impulsion du Secrétariat exécutif du SMSI, la société civile s'est effectivement dotée d'un mécanisme, le Bureau international de la société civile (BISC), lui permettant d'organiser sa représentation dans le débat.

Diverses dispositions ont été mises en place pour permettre à la société civile et au secteur privé de faire des propositions, de les défendre oralement devant les gouvernements, d'assister aux débats et aux discussions en plénière afin de mieux comprendre les positions des uns et des autres.

Le Bureau de la Société Civile confère à la société civile une légitimité interne et assure la représentativité de toutes les familles de la société civile. La première rencontre entre les représentants des deux bureaux (société civile et gouvernements) fut non seulement une première dans 1'histoire des Nations unies, mais aussi un événement exceptionnel que tous les participants ont qualifié d'historique.

Le Bureau m'apparaît comme un instrument très prometteur parce que voulu dès le départ au service de la participation des entités du terrain, et pas seulement comme une structure rassemblant des individus. Le sens même du mot «famille» est là. C'est pourquoi les 16 familles du Bureau sont importantes. Elles représentent la diversité des regards que la société civile porte sur la société de demain: regard de l'universitaire, du spécialiste de la haute technologie, de l'ONG, des jeunes, des médias, des syndicats, des femmes, des populations autochtones, des think tanks, etc. Que la représentativité régionale soit également prise en compte par le Bureau de la Société Civile est également déterminant pour confronter les visions du monde qui varient selon les cultures.

Le BISC nécessite certes d'être consolidé comme canal d'expression des aspirations de la société civile sur tous les sujets d'intérêt global énumérés dans la Déclaration du millénaire. En permettant à la société civile de proposer, de manière concertée, les principes et les actions qu'elle juge les plus appropriés pour répondre aux grands enjeux actuels, le BISC assume déjà un rôle indispensable dans la création de relais, au niveau mondial, entre les espaces de 
débats internationaux et les acteurs de terrain. Ce faisant, sa création constitue une étape fondamentale dans le processus de construction d'un partenariat dynamique, au niveau international, entre gouvernements, société civile, secteur privé et organisations intergouvernementales. Un tel partenariat, prélude à une nouvelle gouvernance mondiale, représente l'une des spécificités les plus significatives du Sommet.

Dans la mesure où le débat entamé a favorisé une prise de conscience par tous les acteurs de l'ensemble des enjeux, on peut considérer, à huit mois de l'échéance - la première phase du Sommet à Genève en décembre 2003 -, que les résultats sont déjà très encourageants.

\section{Des perspectives ouvertes}

Le Sommet lui-même, à Genève comme à Tunis, ne constitue pas une fin en soi, certes, mais il est l'ébauche d'un processus qui permettra, sans nul doute, d'énoncer des principes forts et de dégager des perspectives pour répondre au développement d'une société plus équilibrée, plus solidaire et plus conforme aux principes éthiques dont l'ensemble des acteurs se réclament: une société de la connaissance et des savoirs partagés, fondée sur plus d'écoute mutuelle et de solidarité, au service d'un monde de Paix, grâce au dialogue fécond des cultures et des civilisations.

Pour approfondir le débat et ancrer les résolutions dans le terrain de l'action, il faudra encore du temps et de la patience. En s'appuyant sur les acquis de la première phase du sommet, la société civile a de fortes chances de faire prévaloir, de manière consensuelle et dans un esprit partenarial, ses positions, dont le bien fondé a été largement reconnu.

Construire ensemble, en dépit des difficultés que nous ne manquerons pas de rencontrer, c'est la belle aventure humaine qui nous unit. 